OPEN ACCESS

Edited by:

Hansen Wang

University of Toronto, Canada

Reviewed by:

Knut Holthoff,

University of Jena, Germany

Greg Stuart,

Australian National University,

Australia

Srdjan D. Antic,

University of Connecticut Health

Center, USA

*Correspondence:

Sunggu Yang

sungguyang@inu.ac.kr

Sungchil Yang

sungchil.yang@cityu.edu.hk

Received: 18 June 2016 Accepted: 16 September 2016 Published: 30 September 2016

Citation:

Yang S, Santos MD, Tang C-M,

Kim JG and Yang S (2016)

A Postsynaptic Role for Short-Term

Neuronal Facilitation in

Dendritic Spines.

Front. Cell. Neurosci. 10:224.

doi: 10.3389/fncel.2016.00224

\section{A Postsynaptic Role for Short-Term Neuronal Facilitation in Dendritic Spines}

\author{
Sunggu Yang ${ }^{1 *}$, Mariton D. Santos ${ }^{2,3}$, Cha-Min Tang ${ }^{2}$, Jae Geun Kim ${ }^{4}$ and Sungchil Yang ${ }^{5 *}$ \\ ${ }^{1}$ Department of Nano-Bioengineering, Incheon National University, Incheon, South Korea, ${ }^{2}$ Department of Neurology and \\ Department of Physiology, University of Maryland School of Medicine, Baltimore, MD, USA, ${ }^{3}$ Office of Dietary Supplement \\ Programs, Center for Food Safety and Applied Nutrition, US Food and Drug Administration, College Park, MD, USA, \\ ${ }^{4}$ Division of Life Sciences, College of Life Sciences and Bioengineering, Incheon National University, Incheon, South Korea, \\ ${ }^{5}$ Department of Biomedical Sciences, City University of Hong Kong, Kowloon, Hong Kong
}

Synaptic plasticity is a fundamental component of information processing in the brain. Presynaptic facilitation in response to repetitive stimuli, often referred to as pairedpulse facilitation (PPF), is a dominant form of short-term synaptic plasticity. Recently, an additional cellular mechanism for short-term facilitation, short-term postsynaptic plasticity (STPP), has been proposed. While a dendritic mechanism was described in hippocampus, its expression has not yet been demonstrated at the levels of the spine. Furthermore, it is unknown whether the mechanism can be expressed in other brain regions, such as sensory cortex. Here, we demonstrated that a postsynaptic response can be facilitated by prior spine excitation in both hippocampal and cortical neurons, using 3D digital holography and two-photon calcium imaging. The coordinated action of pre- and post-synaptic plasticity may provide a more thorough account of information processing in the brain.

Keywords: synaptic integration, sensory perception, dendritic spike, NMDA receptor, short-term synaptic plasticity

\section{INTRODUCTION}

Prior information is retrieved and read out while being integrated with incoming signals (Larson and Lynch, 1986; Körding and Wolpert, 2004; Köver and Bao, 2010; Hasselmo and Stern, 2014; Howard et al., 2014). The dynamic modification of the incoming signals in the brain relies heavily on past experience. In fact, a response facilitation in a temporal sequence is common in the in vivo condition (Calford and Semple, 1995; Brosch and Schreiner, 1997, 2000; Rosen and Mooney, 2003; Wehr and Zador, 2005; Kuo and Wu, 2012). Paired-pulse facilitation (PPF) observed with in vitro brain slices has been suggested as a potential synaptic mechanism of in vivo response facilitation in a sequence (Brosch and Schreiner, 2000; Regehr, 2012). In PPF, a prior electrical pulse enhances a neuronal response to a following pulse as a result of enhanced probability of glutamate release in the presynaptic terminal; thus PPF largely involves a presynaptic role in this response facilitation.

Even before presynaptic PPF was conceptualized, the effect of a prior input on postsynaptic responses had been investigated (Larson and Lynch, 1986). This study suggested that NMDA receptors (NMDARs) could cause a prolongation of the postsynaptic response 
when membrane depolarization precedes synaptic input. Recently, the NMDAR-dependent mechanism for response facilitation has been revisited in hippocampus, namely shortterm postsynaptic plasticity (STPP or dendritic hold and read; Santos et al., 2012; Yang et al., 2014). Upon synaptic input, the "glutamate-bound but $\mathrm{Mg}^{2+}$ blocked" state of postsynaptic NMDARs persists for a period in individual dendrites. The prior information stored in this electrically silent or weak state (or "priming") can then be conditionally read out as a regenerative dendritic spike triggered (or "gated") by subsequent neuronal excitability. Such a mechanism holds two characteristics critical for effective signal processing: (1) it is a biophysical mechanism whereby information of prior glutamate exposure can be stored for short periods of time; and (2) it is a cellular mechanism for robust signal amplification.

Despite the potential significance of such STPP for information processing, it's precise mechanism and subcellular localization has not been adequately demonstrated at the level of the dendritic spine of cortical neurons. Here, we used various activation paradigms and imaging tools in brain slices to demonstrate a postsynaptic role of short-term synaptic plasticity in dendritic spines. These findings help establish a more complete understanding of synaptic integration and plasticity in sensory perception.

\section{MATERIALS AND METHODS}

\section{Brain Slice Preparation}

All animal handling procedures were approved by the Institutional Animal Care and Use Committee of the University of Maryland, Incheon National University, and City University of Hong Kong. Animals were treated in accordance with the National Institutes of Health Guide for the care and use of laboratory animals, and the Animal Welfare Act (7 U.S.C. et seq.). Sprague-Dawley rats and C57BL/6 mice (postnatal age: 3-6 weeks) for brain slices were deeply anesthetized with halothane. The brains were quickly removed and placed into chilled $\left(4^{\circ} \mathrm{C}\right)$, oxygenated (5\% $\mathrm{CO}_{2}$ and $95 \% \mathrm{O}_{2}$ ) slicing medium containing (in $\mathrm{mM}$ ): 212 sucrose, $5 \mathrm{KCl}, 1.23 \mathrm{NaH}_{2} \mathrm{PO}_{4}, 26 \mathrm{NaHCO}_{3}, 11$ glucose, $1.5 \mathrm{MgCl}_{2}, 2.5 \mathrm{CaCl}_{2}$. Transverse slices $(300 \mu \mathrm{m})$ were cut normal along the septo-temporal plane. Brain slices was then transferred to a holding chamber containing oxygenated physiological saline made up of (in $\mathrm{mM}$ ): $124 \mathrm{NaCl}, 4 \mathrm{KCl}$, $1.23 \mathrm{NaH}_{2} \mathrm{PO}_{4}, 26 \mathrm{NaHCO}_{3}, 10$ glucose, $1.5 \mathrm{MgCl}_{2}, 2 \mathrm{CaCl}_{2}$. Also, brain slices of primary auditory cortex were prepared according to our established method (Yang et al., 2011b). After $\sim 1 \mathrm{~h}$ recovery, individual slices were transferred to a recording chamber. Oxygenated physiological saline was continuously superfused at a rate of $1.5 \mathrm{ml} / \mathrm{min}$ at $32-33^{\circ} \mathrm{C}$ temperature.

\section{Whole-Cell Patch Recording}

Whole-cell patch recordings were obtained using an Axon instruments Axoclamp 700B Amplifier (Molecular
Devices), and recording pipettes had tip resistances of 3-7 M $\Omega$ when filled with a solution containing (in $\mathrm{mM}): 135$ K-gluconate, $5 \mathrm{KCl}, 1 \mathrm{MgCl}_{2}, 0.02 \mathrm{CaCl}_{2}$, 0.2 EGTA, 10 HEPES, $4 \mathrm{Na}_{2}$-ATP, $0.3 \mathrm{Na}$-GTP. Alexa 594 $(50 \mu \mathrm{M})$ was included in the internal solution for visualization. The $\mathrm{pH}$ and osmolarity of intracellular solution were adjusted to 7.3 and 290 mOsm, respectively. pClamp Version 10.2 software (Molecular Devices) or Igor Pro (WaveMetrics) was used for data acquisition. Hyperpolarizing current pulses (10-20 pA; 0.5 s duration; 5-10 s interval) were continuously applied to monitor cells' input resistance (Rin). During recordings, the access resistance was often monitored; it was typically 20-30 M $\Omega$. Recordings were excluded if Rin changed by $>15 \%$.

\section{D Digital Holography Uncaging}

The procedures for digital holographic photolysis have been described (Yang et al., 2011a). The holographic beam was brought into the optical axis of an upright fluorescence microscope (Olympus BX51) below the epi-fluorescence unit, with a long-pass dichroic mirror. The output beam of a $150 \mathrm{~mW}$, $405 \mathrm{~nm}$ diode laser (CNI Laser) was expanded by a beam expander $(3 \times)$ to fill the short axis of a reflective spatial light modulator (SLM; LCOS Hamamatsu, model X10468-05). The SLM plane was projected onto the back aperture of the microscope objective through a telescope $(\mathrm{L} 1, \mathrm{f} 1=750 \mathrm{~mm}$; $\mathrm{L} 2$, $\mathrm{f} 2=500 \mathrm{~mm})$. The magnification of the telescope was chosen in order to match the SLM short axis with the diameter of the objective's back aperture (Olympus, $60 \times$, W 0.9 NA). The undiffracted component (zero order spot) was removed by placing a small $(<0.5 \mathrm{~mm})$ anodized metal plate on antireflective coated glass plate at the focal plane of L1. The algorithm for the phase hologram calculation and calibration of the temporal spatial resolution were previously described (Yang et al., 2011a). MNI-caged-L-glutamate was prepared fresh each day at final concentration in physiological solution. Glutamate uncaging pulse duration was set to $0.5 \mathrm{~ms}$. Tetrodotoxin (TTX; $1 \mu \mathrm{M}$ ) was added to examine synaptic responses over a wider range of input intensity except when investigating a role for intrinsic excitability as a gating signal.

The input-output curve of gating and priming responses was tested before STPP experiments as previously established (Santos et al., 2012). The proper intensity of a gating signal was determined based on the input-output curve of gating responses. The laser power (or duration) just before laser intensity giving rise to a dendritic spike was chosen for gating intensity (Figure 1A, 1.3 1.4 $\mathrm{ms}$ in this case). Once a dendritic spike occurred, its amplitude usually became saturated. The laser power of priming intensity was adjusted into a level to potentially induce a dendritic spike upon the given gating input. A wide range of priming intensity $(0.6 \sim 1.2 \mathrm{~ms}$ in this case) produced the read-out response efficiently (Figure 1B). This scheme was also applied to experiments with two-photon imaging. 

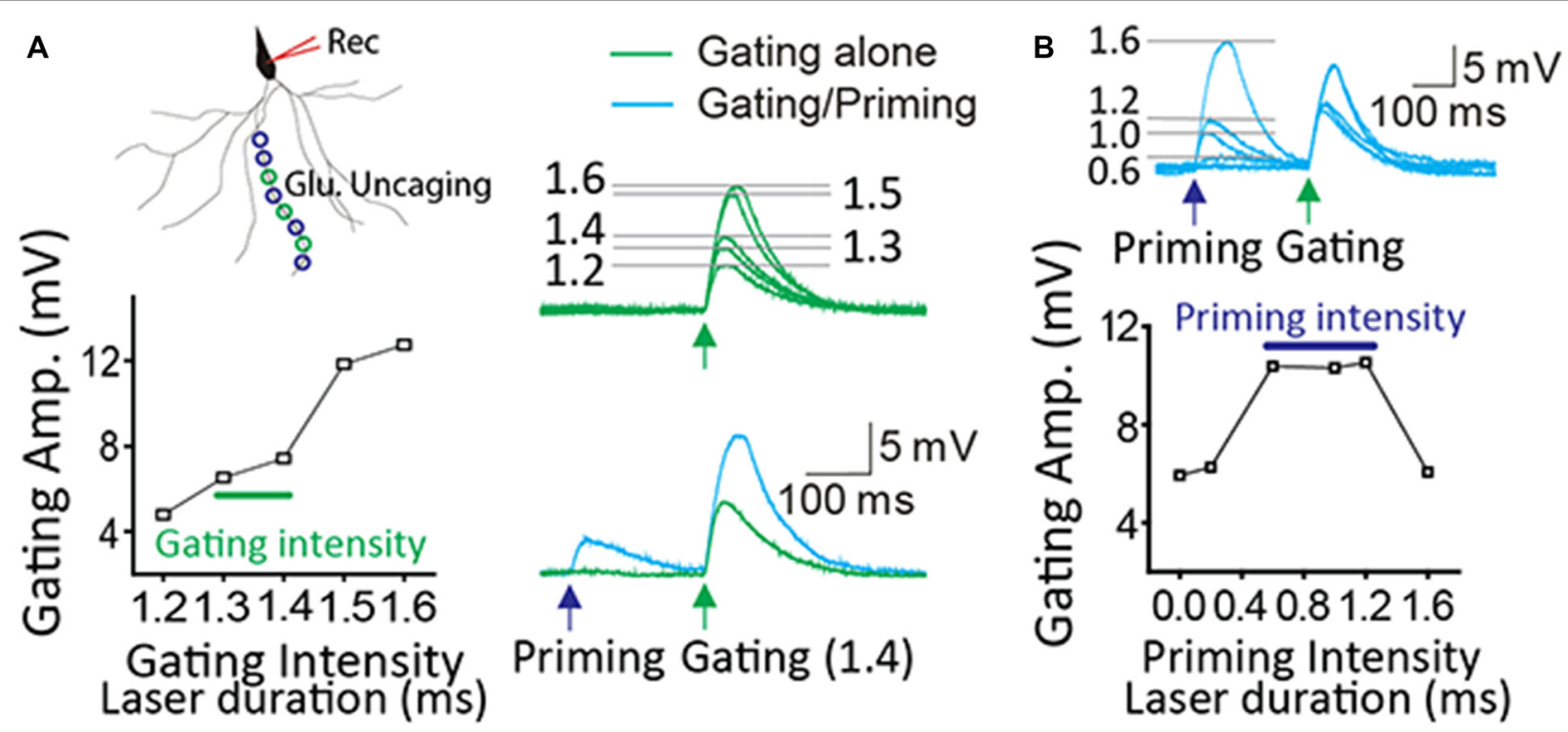

FIGURE 1 | Optimization of priming and gating intensity. (A) The priming stimulation produced the potentiated read-out response when gating stimulation was set up just prior to a dendritic spike. (B) There are a certain range of priming signals to generate a read-out response efficiently. In this case, priming intensity (0.6 1.2 ms laser duration) produced the facilitation when gating intensity ranged from 1.3 to $1.4 \mathrm{~ms}$.

\section{Two-Photon Imaging}

A two-photon imaging system (Prairie Technologies) was used as described previously (Yang et al., 2014, 2016). Briefly, A Chameleon Ultra II laser (Coherent) was tuned to $810 \mathrm{~nm}$ for $\mathrm{Ca}^{2+}$ imaging. Epi- and trans-fluorescence signals were captured through a $60 \times, 1.4 \mathrm{NA}$ oil immersion condenser (Olympus). Fluorescence was split into red and green channels using dichroic mirrors and band-pass filters (T560LPXR, ET525/50, ET620/60; Chroma). Green fluorescence (G, Fluo-5F) was captured on an H10770PA-40 photomultiplier tube (PMTs, Hamamatsu). Red fluorescence (R, Alexa 594) was captured with an R9110 PMT. Data were presented as averages of 10 events per site, and expressed as $\Delta(\mathrm{G} / \mathrm{R}) /(\mathrm{G} / \mathrm{R})$ sat ${ }^{*} 100$ (simply, $\left.\Delta G / G_{\text {sat }}\right)$, where $(G / R)_{\text {sat }}$ was the maximal fluorescence in saturating $(2 \mathrm{mM}) \mathrm{Ca}^{2+}$. The other laser split from a $\mathrm{Ca}^{2+}$ imaging laser path was tuned to $720 \mathrm{~nm}$ for uncaging of MNI-caged-L-glutamate. Glutamate uncaging pulse duration was set to $0.5 \mathrm{~ms}$. All experiments were done at $\sim 32.5^{\circ} \mathrm{C}$ temperature.

\section{Chemicals}

Fluo-5F and Alexa Fluor 594 were from Molecular Probes. MNI-caged-L-glutamate, Ifenprodil, Ro 25-6981 and TTX were purchased from Tocris (Ellisville, MO). NVP-AAM077 was from Sigma. Imaging dyes and MNI-caged-L-glutamate/blockers were introduced to the pipette and the artificial cerebrospinal fluid, respectively.

\section{Statistics}

All data are shown as mean \pm standard error (SEM). An analysis of variance (ANOVA) of Fisher's PLSD post hoc test was performed for between-group comparison, while a paired $t$-test was done for within-groups (significance, ${ }^{*} P<0.05$; ** $P<0.01)$.

\section{RESULTS}

\section{STPP on a Hippocampal Basal Dendrite}

In order to demonstrate STPP on a single basal dendrite, whole cell patch recordings were made on CA1 neurons in hippocampal slices. Alexa $594(20 \mu \mathrm{M})$ in the patch electrode was dialyzed into the dendritic arbor. When a fluorescence signal of a basal dendrite became visible, photolysis sites on spine areas of the dendrite were identified and targeted according to our established method (Yang et al., 2014, 2015). Two sets of photo-stimulation using 3D digital holography were employed to a single dendrite separated temporally and spatially. TTX and MNI-caged-L-glutamate were employed to identify a postsynaptic component of synaptic responses. A priming signal was created by glutamate uncaging to multiple synaptic locations over $\sim 100 \mu \mathrm{m}$ length (blue circles and cyan trace on the blue arrow, Figure 2A). Subsequently, a gating stimulus was directed at neighboring spots on the same dendrite $200 \mathrm{~ms}$ later (green circles and trace on the green arrow, Figure 2A). The gating response was significantly facilitated when preceded by a priming stimulus in all six cells tested (Figure 2A, gating: $3.61 \pm 0.30 \mathrm{mV}$ vs. gating + priming: $6.83 \pm 0.25 \mathrm{mV}$, paired $t$-test, $p<0.001, n=6$ cells). Such facilitation was completely blocked by the application of an NMDAR antagonist, AP5 (1 $\mu \mathrm{M}$ ), an NR2B (an NMDAR subunit) antagonist, Ifenprodil $(1 \mu \mathrm{M})$ and Ro $256981(1 \mu \mathrm{M})$, but not an NR2A antagonist, NVP-AAM077 (0.5 $\mu \mathrm{M})$ which 

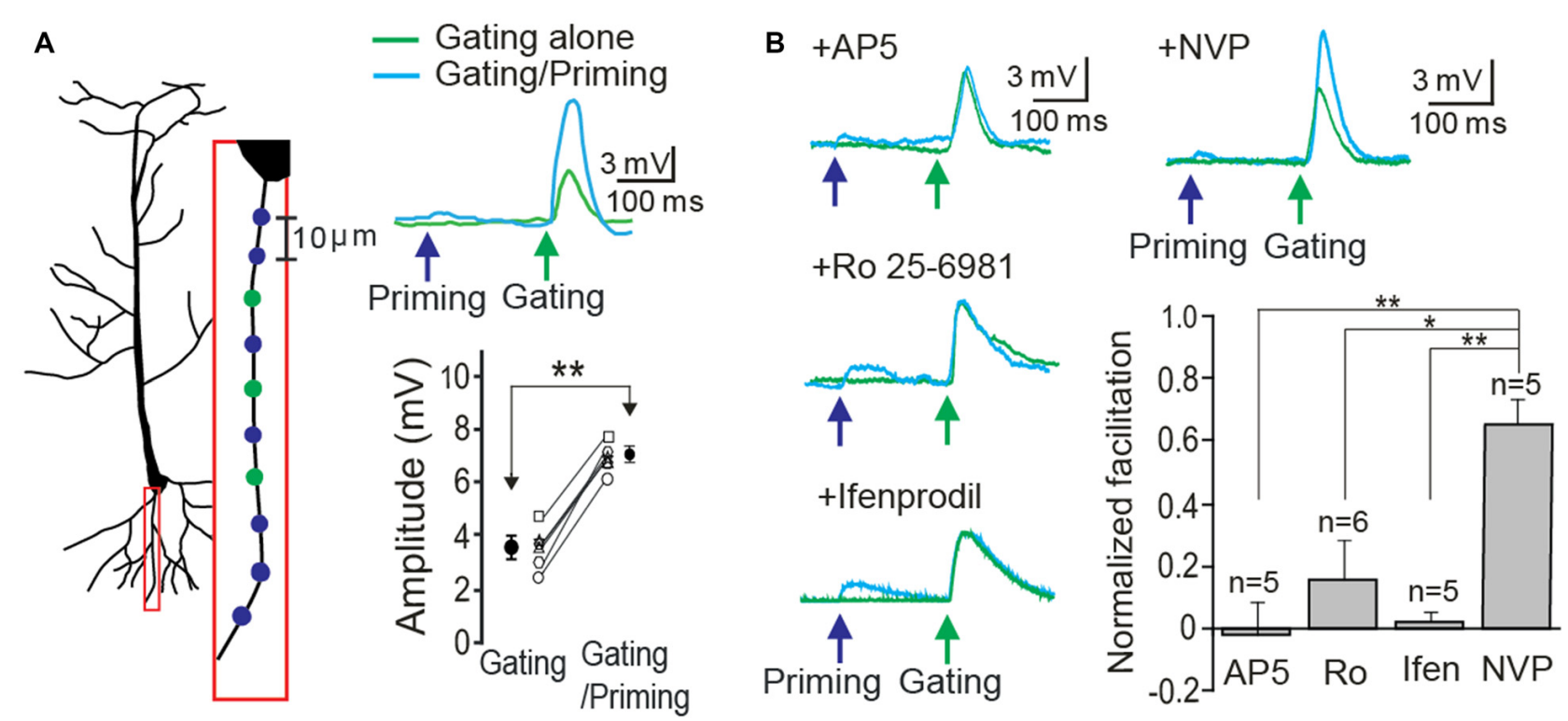

FIGURE 2 | Short-term postsynaptic plasticity (STPP) on a hippocampal dendrite. (A) Illustration of a CA1 pyramidal neuron and priming (blue)/gating (green) glutamate uncaging spots in a basal dendrite (left). The representative responses to priming plus gating (cyan trace) and gating alone (green trace) glutamate uncaging were shown as photoactivated excitatory postsynaptic potentials (EPSPs). Read-out facilitation was observed when a priming photolysis proceeded a gating photolysis ( $n=6$ cells). (B) Neuronal facilitation was completely blocked by AP5 (an NMDAR blocker), Ro 25-6981 and Ifenprodil (NR2B blockers), while it remained by NVP-AAM077 (an NR2A blocker). Error bars represent SEM. ${ }^{*} p<0.05 ; * * p<0.01$.

was known to preferentially block the NR2A subunit of NMDARs at this concentration (Frizelle et al., 2006; Bartlett et al., 2007). With the same gating intensity, facilitation under various antagonists was normalized with that of each control case (Figure 2B, AP5: $-0.02 \pm 0.10$; Ro 25-6981: $0.16 \pm 0.13$; Ifenprodil: $0.02 \pm 0.03 ;$ NVP-AAM077: $0.66 \pm 0.10$, Fisher's PLSD Post hoc test, ${ }^{*} p<0.05$; $\left.{ }^{* *} p<0.01\right)$. This result confirmed our previous demonstration that postsynaptic facilitation can largely occur through an NR2B-dependent mechanism.

Next, we asked whether, in addition to synaptic input, membrane excitability can serve as a gating signal. A single sinusoidal wave for mimicking membrane fluctuation was elicited by injecting a biased sine wave current, while a priming signal was photostimulated on a basal dendrite (Figure 3A). Action potentials (APs) were triggered only when the membrane fluctuation was coupled with the preceded priming input. Then, a gating signal was generated by injecting a sinusoidal current into the soma to mimic an oscillatory sinusoidal wave ( $\sim 8 \mathrm{~Hz}$, a frequent rhythmic form in CA1 hippocampus) while a priming signal was photostimulated on dendritic spines. APs were triggered only when the membrane fluctuation was coupled with the priming signal in the absence of TTX (cyan trace, Figure 3B). They were triggered with $71 \%$ success rate when the photolysis-induced priming signal co-occurred a postsynaptic depolarization (Figure 3B, 5 neurons of 3 animals, AP, 17; no AP, 7). These data suggest that the gating signal can be read out by either synaptic or intrinsic excitability irrespective of the location of input sources.

\section{Hippocampal STPP at the Spine Level}

In order to test the expression of STPP at the spine level, we stimulated individual spines of a CA1 pyramidal neuron using the two-photon calcium imaging method. We identified spines of a basal dendrite that were co-labeled with Alexa $594(20 \mu \mathrm{M})$ and a dynamic $\mathrm{Ca}^{2+}$ indicator Fluo $5 \mathrm{~F}$ (100 $\mathrm{MM}$, Figure 4A). For a priming stimulus, 10 spines of a basal dendrite (blue circles) were targeted for photolysis, while a gating stimulus targeted five of these spines (green outlines). The gating stimulus alone produced an electrical response in the soma (the green trace on the green arrow head, Figure $4 \mathbf{B i}, n=9$ cells) and $\mathrm{Ca}^{2+}$ transients on the spines (the green trace on the green arrow head, 5 cells among 9 cells, Figure $\mathbf{4 C i}$ ). When a priming stimulus preceded the gating stimulus, both the electrical and calcium signals were remarkably facilitated (Figure 4Bii, gating alone: $2.3 \pm 0.35 \mathrm{mV}$ vs. gating/priming: $7.17 \pm 0.73 \mathrm{mV}$, paired$t$ test, $p<0.001, n=9$; Figure 4Cii, normalized $\Delta \mathrm{G} / \mathrm{G}_{\mathrm{sat}}$ of gating alone: $1.78 \pm 0.21$ vs. gating/priming: $2.94 \pm 0.20$, paired- $t$ test, $p<0.001, n=5$ ). This result suggests that STPP is a response integrated by dynamic activity of individual spines.

\section{Cortical STPP}

Next we asked whether STPP is present in other brain areas. To this end, layer 4 pyramidal neurons of primary auditory cortex were tested with the same experimental strategy as CA1 pyramidal neurons. Ten spines of a cortical neuron were identified and targeted to photolysis of MNI- 

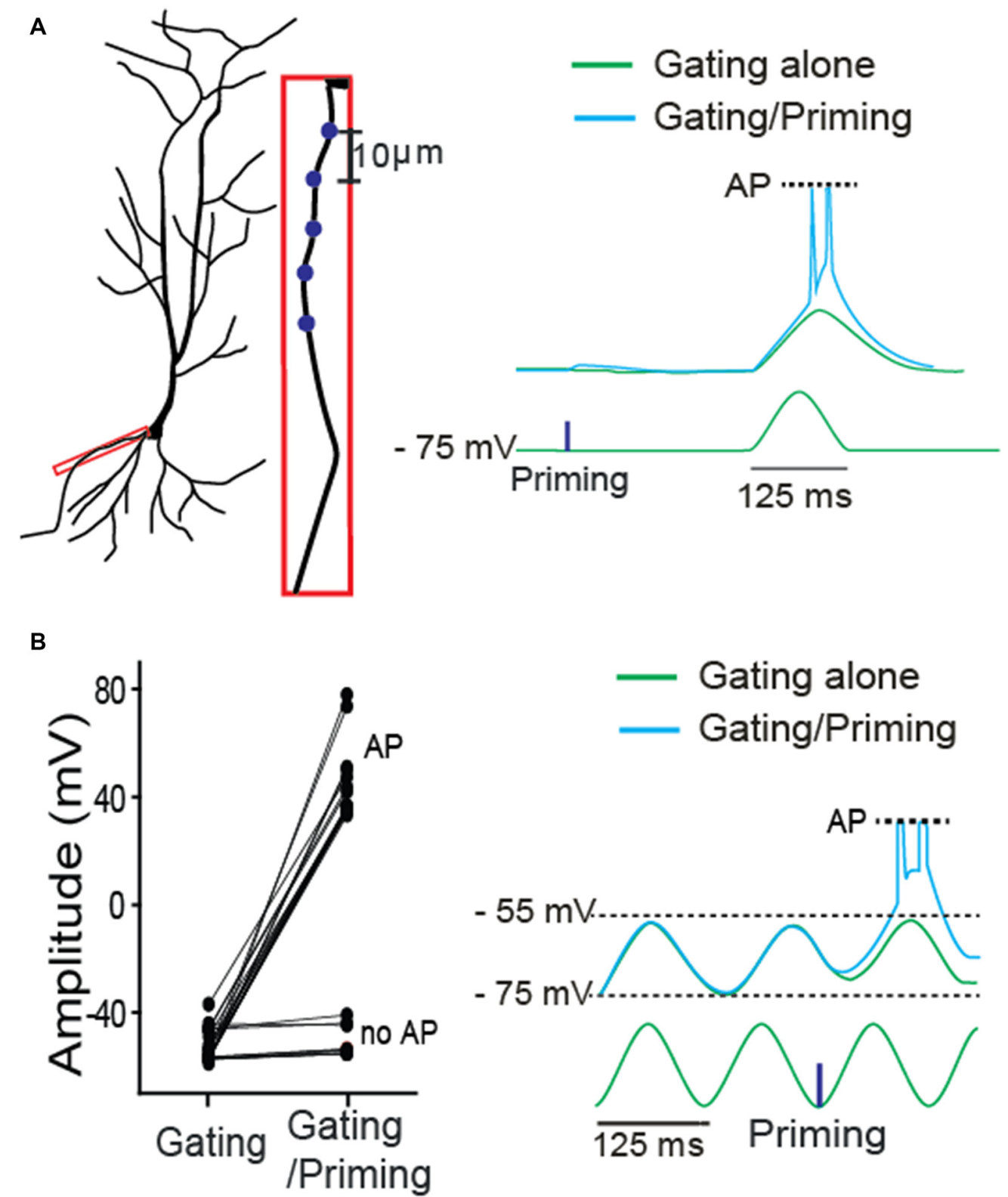

FIGURE 3 | A role of intrinsic membrane excitability as a gating signal in STPP. (A) Blue dots for priming photostimulation were positioned in a basal dendrite. The read-out action potentials (APs) were observed only when intrinsic excitability was combined with the prepositioned priming photolysis. Blue dots for priming photostimulation were located in a basal dendrite. (B) $\sim 8 \mathrm{~Hz}$ oscillatory sinusoidal wave elicited by current injection triggered APs only when the peak was preceded by a priming signal (uncaging). The all-or-none APs were shown with 71\% success rate (AP: $n=17 ;$ no AP: $n=7$ ).

glutamate (Figure 5A). Similar to the results from hippocampal neurons, the electrical responses in the soma were remarkably facilitated when a priming stimulus preceded the gating stimulus (Figures 5Bi,ii, gating alone: $3.3 \pm 0.35 \mathrm{mV}$ vs. gating/priming: $4.86 \pm 0.62 \mathrm{mV}$, paired $t$ test, $p<0.001$, $n=12$ ). The facilitation was completely blocked by the application of an NR2B selective blocker, Ifenprodil (1 $\mu \mathrm{M}$; Figures 5Ci,ii, gating alone: $3.10 \pm 0.63 \mathrm{mV}$ vs. gating/priming: $3.20 \pm 0.59 \mathrm{mV}$, paired $t$ test, $p>0.05$, $n=5)$. These results implicate that NMDAR-dependent activity within individual spines mediate STPP in cortical neurons.

\section{DISCUSSION}

In the past, studying STPP has been difficult due to limitation in recording techniques. With the advent of biochemical activation and imaging tools including $3 \mathrm{D}$ digital holography and two- 

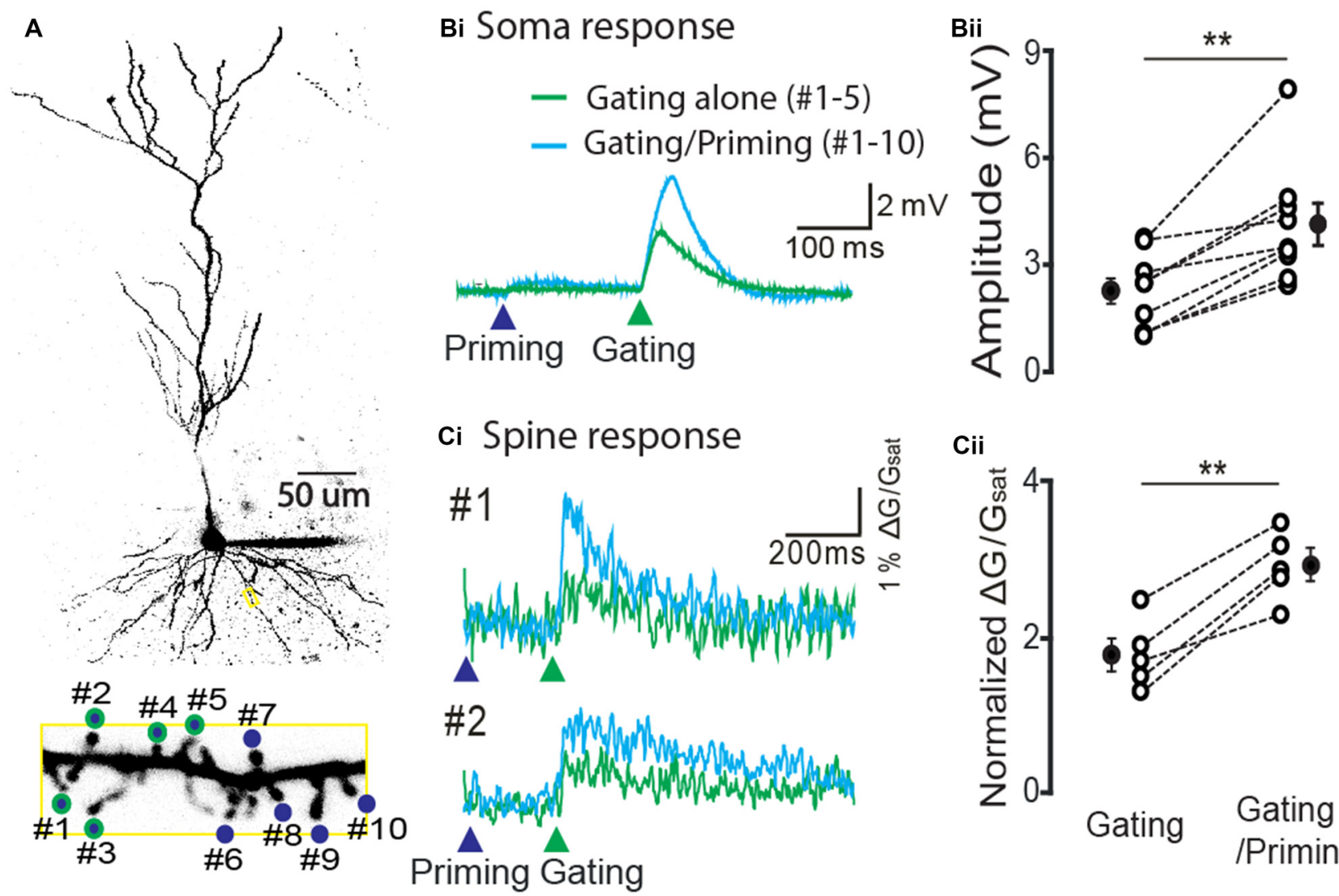

FIGURE 4 | Hippocampal STPP at a spine level. (A) Two photon image of a CA1 pyramidal neuron and its basal dendrite with priming (blue color)/gating glutamate uncaging spots (green outline) on the individual spines in a basal dendrite. (B,C) The representative responses to priming plus gating (cyan trace) and gating alone (green trace) glutamate uncaging were shown as photoactivated EPSPs. When the priming stimulus proceeded the gating stimulus, the facilitated read-out responses (cyan traces) were elicited in both the electrical recording at the cell body (Bi) and calcium transients on individual spines (Ci). Population data in electrical recording on cell body (Bii, $n=9$ cells $/ 4$ animals) and calcium transients on the spines (Cii, $n=5$ cells/3 animals). Error bars represent SEM. ${ }^{* *} p<0.01$.

photon microscopy, we demonstrate here the existence of STPP in hippocampus and sensory cortex. The information storage mechanism is present in dendritic spines. Such information held for a short period of time can facilitate gating signals such as fluctuations in synaptic and intrinsic excitability, suggesting the important role on sensory signal processing.

\section{The Role of STPP in Sensory Information Processing}

Response facilitation in a temporal sequence has been previously suggested as a core model of feature detection and discrimination (Calford and Semple, 1995; Brosch and Schreiner, 1997, 2000; Fortune and Rose, 2000; Wehr and Zador, 2005; Schreiner and Polley, 2014). PPF tested with in vitro brain slices has been suggested as a potential synaptic mechanism of the observed in vivo response facilitation (Brosch and Schreiner, 2000). However, the causal relationship between in vitro $\mathrm{PPF}$ and in vivo response facilitation still remains speculative. It is noteworthy that the NMDAR-dependent STPP is similar to the in vivo response enhancement phenomenon in light of the temporal scale ( $600 \mathrm{~ms}$ ) and all-or-none expression pattern (Brosch and Schreiner, 2000; Yang et al., 2014). Hence, we propose that STPP acts along with PPF as a cellular process underlying sensory perception.

\section{Prerequisites for STPP}

STPP is determined by a combination of several factors such as dendritic morphology, the spatiotemporal pattern of synaptic inputs and active intrinsic conductances. STPP was observed when a priming and gating stimulus were co-localized on the same distal dendrite. By contrast, it was not observed when one of two stimuli was separately directed on an apical trunk or adjacent dendrite and two inputs were separated by more than $50 \mu \mathrm{m}$ even on the same dendrite (Santos et al., 2012). At the spine level, however, we showed that priming and gating synaptic inputs can be neighboring ones for STPP induction as in Figure 2A. Also, a gating stimulus of rhythmic membrane depolarization (which does not necessarily share the same synapses with the priming stimulus) consistently induced STPP, showing independence of input sources (Figure 3). It is notable that different input patterns of a gating stimulus, whether spatially distributed or 
$\mathbf{A i}$

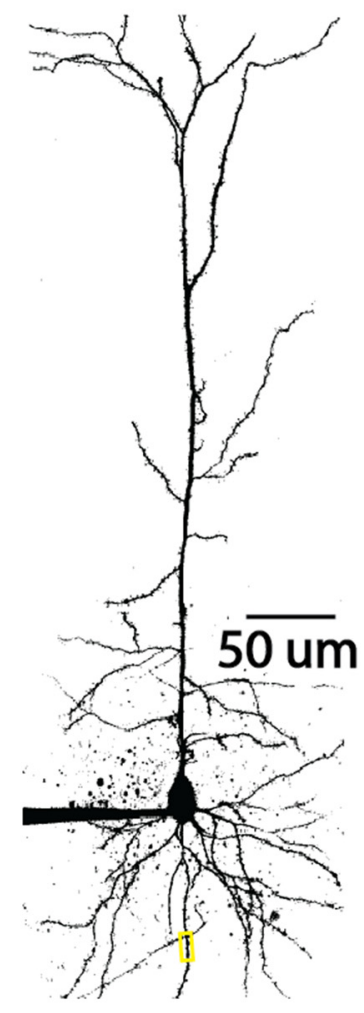

Aii

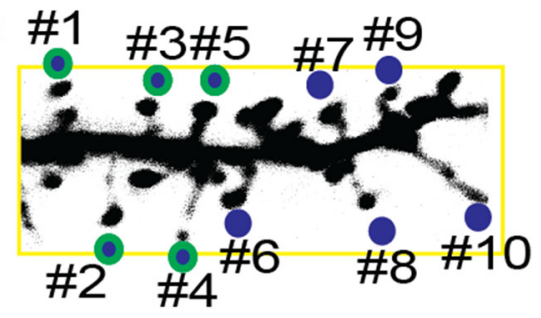

Bi Soma response

- Gating alone (\#1-5)

- Gating/Priming (\#1-10)

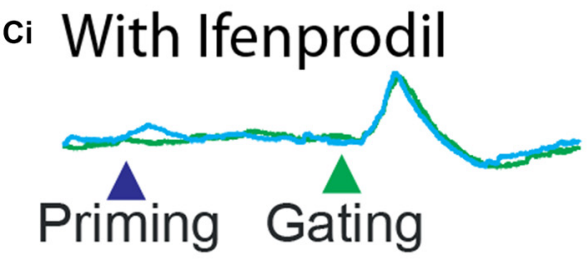

Bii Soma response

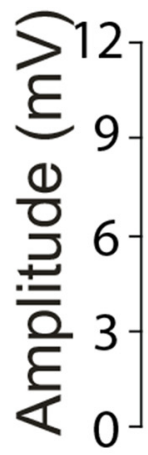

Cii Soma response
(with Ifenprodil)

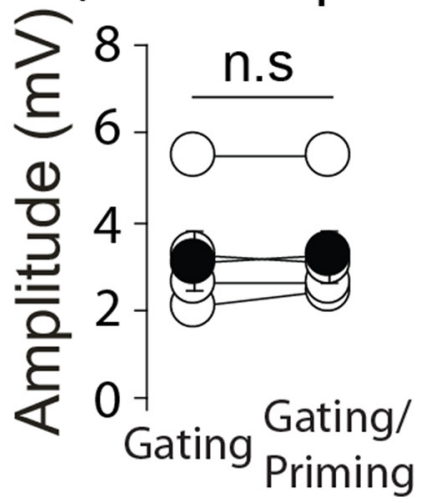

FIGURE 5 | Cortical STPP. (A) Two photon image of a pyramidal neuron (Ai) and its basal dendrite with priming (blue color)/gating (green outline) glutamate uncaging spots on the individual spines in a basal dendrite (Aii). (B) The representative responses to priming plus gating (cyan trace) and gating alone (green trace) glutamate uncaging were shown as photoactivated EPSPs. When the priming stimulus preceded the gating stimulus, the facilitated response (cyan trace) was elicited in the electrical recording at the cell body (Bi). (C) NR2B selective blocker, Ifenprodil, completely blocked the neuronal facilitation (Ci). Population data of control (Bii, $n=12$ cells/4 animals) and Ifenprodil (Cii, $n=5$ cells/4 animals) in electrical recording of the cell body. ${ }^{* *} p<0.01 ; n . s, P>0.05$.

clustered within a single dendrite, were capable of producing facilitation, which is consistent with a previous study (Losonczy and Magee, 2006). Taken together, these data provide evidence that facilitation likely occurs only when a priming and gating input are situated on the same dendrite unless they are not far apart from each other within the dendrite. Consistent with our findings, there is physiological evidence that single dendrites are tuned to different orientations of object movement in pyramidal neurons of visual cortex (Jia et al., 2010).

STPP can be induced by its characteristic regenerative $\mathrm{Ca}^{2+}$ influx through NMDARs (i.e., NMDA spikes). Although the basal level of a synaptic event is largely initiated by nonNMDAR-mediated cationic currents, its amplification begins with NMDA spikes in thin dendrites where most excitatory synaptic events occur. An earlier synaptic input ("priming stimulus") may not produce a depolarization large enough to remove the $\mathrm{Mg}^{2+}$ block of the NMDARs. However, when a subsequent stimulus ("gating stimulus") which by itself would produce only a modest depolarization is delivered to the same dendrite, its response can be markedly facilitated by promoting $\mathrm{Mg}^{2+}$ liberation and initiating an NMDA spike. The question then arises whether STPP can be a false-positive facilitation due to prolonged dendrite depolarization following a priming input (Milojkovic et al., 2004). However, the $200 \mathrm{~ms}$ interval between two stimuli, in our study, was sufficient to prevent voltage and calcium deflections from overlapping. Priming responses were fully recovered into baseline as shown in both somatic recording and spine calcium imaging (Figure 4). Also, STPP was induced even at longer intervals up to $600 \mathrm{~ms}$ which could guarantee temporal separation (Yang et al., 2014). These results support the STPP mechanism of the "glutamate-bound but $\mathrm{Mg}^{2+}$ blocked" state rather than electrical interference by prolonged dendritic depolarization. Those physiological characteristics of STPP are distinct from those of the PPF with respect to location of action (PPF, presynaptic glutamate release vs. STPP, postsynaptic NMDARs), activation pattern (PPF, graded vs. STPP, NMDA spike-driven all-or-none) and spatial integration spectrum (PPF: synapse-specific vs. STPP, dendrite-specific). 


\section{The Neuronal Structure for STPP}

Pyramidal neurons are connected to one another in an associational fashion along the longitudinal axis (e.g., CA1CA1 connection) in hippocampus (Yang et al., 2014) and also along the representation of stimulus features (e.g., isofrequency corticocortical line) in cortex (Imig and Reale, 1980; Lee and Winer, 2008; Oswald and Reyes, 2008; Brown and Hestrin, 2009). The connectivity of the pyramidalpyramidal neurons and their biophysical properties (holding and amplifying electrical traces) allow signals to pass through a linearly connected brain circuit. Considering that neurons receive streams of information in a temporal sequence, the brain must have a capacity to process a temporal sequence of information for a short period of time; the individual information cannot be properly interpreted without a form of short-term mnemonic buffer. Signals bearing temporal information (e.g., priming, including subthreshold responses) can be, for example, read out by pronounced neuronal activity (i.e., gating, including a certain frequency of brain oscillation), and subsequently converted into a spatial pattern of information along the linear circuit (Hasselmo and Stern,

\section{REFERENCES}

Bartlett, T. E., Bannister, N. J., Collett, V. J., Dargan, S. L., Massey, P. V., Bortolotto, Z. A., et al. (2007). Differential roles of NR2A and NR2B-containing NMDA receptors in LTP and LTD in the CA1 region of two-week old rat hippocampus. Neuropharmacology 52, 60-70. doi: 10.1016/j.neuropharm.2006. 07.013

Brosch, M., and Schreiner, C. E. (1997). Time course of forward masking tuning curves in cat primary auditory cortex. J. Neurophysiol. 77, 923-943.

Brosch, M., and Schreiner, C. E. (2000). Sequence sensitivity of neurons in cat primary auditory cortex. Cereb. Cortex 10, 1155-1167. doi: 10.1093/cercor/10. 12.1155

Brown, S. P., and Hestrin, S. (2009). Intracortical circuits of pyramidal neurons reflect their long-range axonal targets. Nature 457, 1133-1136. doi: 10 . 1038/nature07658

Calford, M. B., and Semple, M. N. (1995). Monaural inhibition in cat auditory cortex. J. Neurophysiol. 73, 1876-1891.

Fortune, E. S., and Rose, G. J. (2000). Short-term synaptic plasticity contributes to the temporal filtering of electrosensory information. J. Neurosci. 20, 7122-7130.

Frizelle, P. A., Chen, P. E., and Wyllie, D. J. A. (2006). Equilibrium constants for $(R)$-[(S)- 1-(4-bromo-phenyl)-ethylamino]-(2,3-dioxo-1,2,3,4tetra-hydroquinoxalin-5-yl)-methyl]-phosphonic acid (NVP-AAM077) acting at recombinant NR1/NR2A and NR1/NR2B N-methyl-D-aspartate receptors: implications for studies of synaptic transmission. Mol. Pharmacol. 70, 1022-1032. doi: 10.1124/mol.106.024042

Hasselmo, M. E., and Stern, C. E. (2006). Mechanisms underlying working memory for novel information. Trends Cogn. Sci. 10, 487-493. doi: 10.1016/j. tics.2006.09.005

Hasselmo, M. E., and Stern, C. E. (2014). Theta rhythm and the encoding and retrieval of space and time. Neuroimage 85, 656-666. doi: 10.1016/j. neuroimage.2013.06.022

Howard, M. W., MacDonald, C. J., Tiganj, Z., Shankar, K. H., Du, Q., Hasselmo, M. E., et al. (2014). A unified mathematical framework for coding time, space and sequences in the hippocampal region. J. Neurosci. 34, 4692-4707. doi: 10.1523/JNEUROSCI.5808-12.2014

Imig, T. J., and Reale, R. A. (1980). Patterns of cortico-cortical connections related to tonotopic maps in cat auditory cortex. J. Comp. Neurol. 192, 293-332. doi: 10. 1002/cne.901920208

Jia, H., Rochefort, N. L., Chen, X., and Konnerth, A. (2010). Dendritic organization of sensory input to cortical neurons in vivo. Nature 464, 1307-1312. doi: 10. $1038 /$ nature 08947
2006, 2014; Santos et al., 2012). This signal conversion of time-to-space may be necessary for object recognition in the brain.

\section{AUTHOR CONTRIBUTIONS}

Sunggu Yang, MDS, C-MT and Sungchil Yang designed experiments. Sunggu Yang, MDS, JGK and Sungchil Yang performed the experiments and prepared the figures. Sunggu Yang and Sungchil Yang wrote the main manuscript text. All authors reviewed the manuscript.

\section{ACKNOWLEDGMENTS}

We thank Ken Burke for his constructive comments of this article. This work was supported by an Incheon National University (International Cooperative) Research Grant for Sunggu Yang, and a CityU Research Project (project No. 9610347) and center for biosystems, neuroscience and nanotechnology (project No. 9360148) for Sungchil Yang. The authors declare no competing financial interests.

Körding, K. P., and Wolpert, D. M. (2004). Bayesian integration in sensorimotor learning. Nature 427, 244-247. doi: 10.1038/nature 02169

Köver, H., and Bao, S. (2010). Cortical plasticity as a mechanism for storing Bayesian priors in sensory perception. PLoS One 5:e10497. doi: 10.1371/journal. pone.0010497

Kuo, R. I., and Wu, G. K. (2012). The generation of direction selectivity in the auditory system. Neuron 73, 1016-1027. doi: 10.1016/j.neuron.2011. 11.035

Larson, J., and Lynch, G. (1986). Induction of synaptic potentiation in hippocampus by patterned stimulation involves two events. Science 232, 985-988. doi: 10.1126/science. 3704635

Lee, C. C., and Winer, J. A. (2008). Connections of cat auditory cortex: III. Corticocortical system. J. Comp. Neurol. 507, 1920-1943. doi: 10.1002/cne. 21613

Losonczy, A., and Magee, J. C. (2006). Integrative properties of radial oblique dendrites in hippocampal CA1 pyramidal neurons. Neuron 50, 291-307. doi: 10.1016/j.neuron.2006.03.016

Milojkovic, B. A., Radojicic, M. S., Goldman-Rakic, P. S., and Antic, S. D. (2004) Burst generation in rat pyramidal neurones by regenerative potentials elicited in a restricted part of the basilar dendritic tree. J. Physiol. 558, 193-211. doi: 10. 1113/jphysiol.2004.061416

Oswald, A.-M. M., and Reyes, A. D. (2008). Maturation of intrinsic and synaptic properties of layer $2 / 3$ pyramidal neurons in mouse auditory cortex. J. Neurophysiol. 99, 2998-3008. doi: 10.1152/jn.01160.2007

Regehr, W. G. (2012). Short-term presynaptic plasticity. Cold Spring Harb. Perspect. Biol. 4:a005702. doi: 10.1101/cshperspect.a005702

Rosen, M. J., and Mooney, R. (2003). Inhibitory and excitatory mechanisms underlying auditory responses to learned vocalizations in the songbird nucleus HVC. Neuron 39, 177-194. doi: 10.1016/s0896-6273(03) 00357-x

Santos, M. D., Mohammadi, M. H., Yang, S., Liang, C. W., Kao, J. P. Y., Alger, B. E., et al. (2012). Dendritic hold and read: a gated mechanism for short term information storage and retrieval. PLoS One 7:e37542. doi: 10.1371/journal. pone. 0037542

Schreiner, C. E., and Polley, D. B. (2014). Auditory map plasticity: diversity in causes and consequences. Curr. Opin. Neurobiol. 24, 143-156. doi: 10.1016/j. conb.2013.11.009

Wehr, M., and Zador, A. M. (2005). Synaptic mechanisms of forward suppression in rat auditory cortex. Neuron 47, 437-445. doi: 10.1016/j.neuron.2005. 06.009 
Yang, S., Ben-Shalom, R., Ahn, M., Liptak, A. T., van Rijn, R. M., Whistler, J. L., et al. (2016). $\beta$-arrestin-dependent dopaminergic regulation of calcium channel activity in the axon initial segment. Cell Rep. 16, 1518-1526. doi: 10.1016/j. celrep.2016.06.098

Yang, S., Yang, S., Moreira, T., Hoffman, G., Carlson, G. C., Bender, K. J., et al. (2014). Interlamellar CA1 network in the hippocampus. Proc. Natl. Acad. Sci. U S A 111, 12919-12924. doi: 10.1073/pnas.1405468111

Yang, S., Papagiakoumou, E., Guillon, M., de Sars, V., Tang, C.-M., and Emiliani, V. (2011a). Three-dimensional holographic photostimulation of the dendritic arbor. J. Neural Eng. 8:046002. doi: 10.1088/1741-2560/8/4/ 046002

Yang, S., Weiner, B. D., Zhang, L. S., Cho, S.-J., and Bao, S. (2011b). Homeostatic plasticity drives tinnitus perception in an animal model. Proc. Natl. Acad. Sci. U S A 108, 14974-14979. doi: 10.1073/pnas.1107998108
Yang, S., Tang, C.-M., and Yang, S. (2015). The shaping of two distinct dendritic spikes by A-type voltage-Gated $\mathrm{K}^{+}$channels. Front. Cell. Neurosci. 9:469. doi: 10.3389/fncel.2015.00469

Conflict of Interest Statement: The authors declare that the research was conducted in the absence of any commercial or financial relationships that could be construed as a potential conflict of interest.

Copyright (C) 2016 Yang, Santos, Tang, Kim and Yang. This is an open-access article distributed under the terms of the Creative Commons Attribution License (CC BY). The use, distribution and reproduction in other forums is permitted, provided the original author(s) or licensor are credited and that the original publication in this journal is cited, in accordance with accepted academic practice. No use, distribution or reproduction is permitted which does not comply with these terms. 